\section{Internetpolitik in Deutschland}

\author{
von Raymund Werle, Max-Planck-Institut für \\ Gesellschaftsforschung, Köln
}

Die Rahmenbedingungen nationalstaatlicher Technologiepolitik haben sich in den letzten 30 Jahren grundlegend geändert. Trotz der tendenziellen Erosion des klassischen Nationalstaats bestehen aber weiterhin Gestaltungsmöglichkeiten, die auch genutzt werden. In Deutschland war es bis in die zweite Hälfte der 1990er Jahre möglich, eine konzertierte, der deutschen informations- und kommunikationstechnischen Industrie vermeintlich nützliche Politik gegen das TCP/IP basierte Internet zu betreiben. Erst mit der Änderung der institutionellen Rahmenbedingungen des Politikfeldes wurden die Konsequenzen der Fehlsteuerung sichtbar. Die neue, ebenfalls national spezifische Politik trägt der infrastrukturellen Bedeutung des Internets Rechnung. Sie fördert den Zugang zum Netz und die Nutzung des Netzes und versucht, die hierzu notwendigen Rahmenbedingungen auf nationaler und internationaler Ebene zu schaffen. Die Internetpolitik ist inkrementell. Aus unterschiedlichen Quellen wird eine Vielzahl kleinerer Forschungsund Entwicklungsprojekte finanziert.

\section{Einführung}

Vielen erscheint das Internet als der Prototyp einer globalisierten Technik. Aufsetzend auf einer dichten technischen Kommunikationsinfrastruktur funktioniert das Netz mit einer transparenten Architektur und einer relativ kleinen Zahl weltweit akzeptierter technischer Basisprotokolle. Sie lassen auf der Ebene der Dienste vielfältige Möglichkeiten der Anwendung und Gestaltung offen, was Ausschlag gebend ist für die schnelle Ausbreitung des Netzes. Doch sind es nicht nur technische Gründe, die den Erfolg des Internets als einer multifunktionalen Infrastruktur erklären, zumal das Netz sich nicht in allen Ländern, auch nicht in der industrialisierten Welt, gleichermaßen schnell ausbreitet. Auch politische Maßnahmen - von der Industrie- und Wirtschaftspolitik bis zur Wissenschafts- und Infrastrukturpolitik sowie neuerdings der Innovationspolitik - spielen eine Rolle. Um diese auf das Internet gerichteten Politi- ken zusammenfassend zu bezeichnen, soll hier der Begriff der Internetpolitik benutzt werden.

Im Zentrum der folgenden Betrachtung steht mit der nationalen Internetpolitik ein Politikbereich, bei dem gleichermaßen die Steuerbarkeit des Gegenstands und die Steuerungsfähigkeit der Akteure in Frage gestellt werden. Der globale Charakter der Technik einerseits und die ökonomischen und politischen Europäisierungs- und Globalisierungsprozesse andererseits werden in der Regel als die für die Steuerungsprobleme maßgeblichen Ursachen angeführt.

Die Steuerbarkeit des Internets ist seit seiner Entstehung umstritten. Eine Technik ist nicht beliebig formbar, insbesondere dann nicht (mehr), wenn sie sich wie das Internet großflächig ausbreitet. Meist geben Pfadabhängigkeiten einen Korridor vor, in dem sich die Technik entwickelt. So ist es zum gegenwärtigen Zeitpunkt schwer vorstellbar, dass sich ein funktional dem Internet vergleichbares globales Kommunikationsnetz entwickelt, das eine andere Architektur aufweist und auf anderen Basisstandards aufbaut. Gelegentlich wird allerdings die Befürchtung laut, dass die großen Netzbetreiber und Diensteanbieter Änderungen in zentralen Komponenten des Internets vornehmen könnten, die - gewollt oder ungewollt - die Architektur des Netzes schrittweise verändern (Blumenthal und Clark 2001). Solche Änderungen dienen vor allem mit Blick auf kommerzielle Transaktionen dazu, das Internet sicherer zu machen. Derartige Entwicklungen entziehen sich der direkten politischen Steuerung, weil das Internet nicht einem einzigen oder sehr wenigen Netzbetreibern und Diensteanbietern gehört. Zudem lässt sich das Verhalten der unübersehbar großen Zahl von Nutzern eines immer größer werdenden Angebots von Diensten kaum prognostizieren geschweige denn direkt steuern. Dies bedeutet jedoch nicht, wie speziell die amerikanische rechts- und technologiepolitische Diskussion zeigt, dass vor allem indirekte politische Einflussnahme auf das Internet bzw. die Rahmenbedingungen seiner Entwicklung und Nutzung unterbleiben soll (Lemley und Lessig 2004). Es fragt sich allerdings, ob einzelne Staaten mit Ausnahme der USA hierzu in der Lage sind.

Diese Frage bezieht sich auch ganz allgemein auf die Steuerungsfähigkeit der Nationalstaaten. Unbestreitbar haben im Zuge der inter- 
nationalen Arbeitsteilung grenzüberschreitende ökonomische Transaktionen und soziale Beziehungen zugenommen, wurde einzelstaatliches Handeln vielfältig in internationale Normen eingebunden. Mit dem damit einhergehenden Prozess der Liberalisierung verlagerten sich „Steuerungsfunktionen von (nationalstaatlichen) Hierarchien auf (internationale) Märkte“ (Streeck 2004, S. 9). Dieser Prozess ist jedoch das Ergebnis überwiegend nationaler politischer Steuerungs- bzw. Reformmaßnahmen, die auch im Vertrauen auf die innovativen Kräfte des Marktes ergriffen wurden, also innovationspolitisch motiviert waren. Das gilt speziell auch für die Prozesse der Liberalisierung und Privatisierung der großen technischen Infrastruktursysteme wie Eisenbahn, Elektrizitätsversorgung und Telekommunikation. Hier hat sich der Staat aus der Rolle des übermächtigen Steuerungsakteurs, der diese Systeme auch betrieben hat, zurückgezogen. In Deutschland und anderswo bleibt ihm aber die immer noch einflussreiche Rolle des Regulierers und Koordinators dieser Infrastruktursysteme (Werle 2001).

Die Einbettung nationalstaatlichen Handelns in das europäische Mehrebenensystem und eine europäische Verfassung, die vor allem ,negative Integration“, d. h. Marktintegration und Wettbewerb fördert, hat wie die ökonomische Globalisierung den Effekt, staatliche Steuerungsmöglichkeiten einzuschränken (Scharpf 1999). Doch lassen sich in der Forschungs- und Technologiepolitik durchaus auch Beispiele dafür finden, dass die vermeintliche Schwäche zur Stärke wurde (Grande 1996). Es lässt sich sogar argumentieren, dass die Etablierung neuer transnationaler und internationaler technologiepolitischer Handlungsarenen in der EU und in internationalen Regimen die Handlungsfähigkeit des Nationalstaats erweitert (Simonis 2004).

Auch wenn der Nationalstaat insgesamt schwächer geworden ist, wäre es wohl voreilig, zum gegenwärtigen Zeitpunkt von einer Erosion des Nationalstaats und einem weitgehenden Verlust nationaler Steuerungsfähigkeit zu sprechen. Das gilt auch für die Technologiepolitik. Steuerungsfähigkeit garantiert allerdings noch keinen Steuerungserfolg. Dieser wird, wie noch zu zeigen ist, zu einem guten Teil durch die institutionellen Bedingungen beeinflusst, unter denen die Steuerungsakteure handeln. Mit Blick auf eine in die EU-Politik eingebettete, aber nicht von ihr determinierte deutsche Internetpolitik lässt sich sagen, dass trotz der eingeschränkten Steuerungsfähigkeit der Nationalstaaten und der schwierigen Steuerbarkeit des Internets Steuerung möglich und auch notwendig ist. Dabei kann Steuerung gelegentlich auch darin bestehen, dass die Politik darauf verzichtet, Einfluss zu nehmen. So hat zum Beispiel die amerikanische Federal Communications Commission (FCC) über viele Jahre eine Politik der Nichtregulierung des Internets verfolgt, obwohl das Netz Ansatzpunkte für Regulierungseingriffe bietet.

Im Folgenden wird die Entwicklung der deutschen Internetpolitik seit den frühen 1980er Jahren skizziert und im Hinblick auf den Steuerungserfolg bewertet. Besonderes Augenmerk liegt hierbei auf den institutionellen Bedingungen staatlicher Steuerung. Ganz grob lassen sich zwei Perioden der deutschen Internetpolitik unterscheiden. Die erste Periode, die spät in der zweiten Hälfte der 1990er Jahre endete, war geprägt durch eine eng geführte Förderung nationaler oder europäischer Technologien mit dem Ziel der Stärkung der nationalen Champions. In der zweiten Periode dominiert das Ziel der Entwicklung und Modernisierung der nationalen Kommunikationsinfrastruktur mit Hilfe des Internets und der Schaffung adäquater Rahmenbedingungen der Nutzung dieser Infrastruktur.

\section{Eine verfehlte Internetpolitik bis in die zweite Hälfte der 1990er Jahre}

Im Rückblick auf die Entwicklung des Internets gibt es keinen Zweifel, dass die zentralen Vorläufernetze des heutigen weltweit verbreiteten und kommerziell hoch attraktiven Netzes amerikanische Forschungsnetze waren. Diese Netze wurden lange Zeit überwiegend aus öffentlichen Mitteln finanziert. Sie waren Teil einer Politik, die sowohl die Infrastruktur der Datenkommunikation im amerikanischen Wissenschafts- und Forschungssektor ausbauen als auch die auf diesen Bereich bezogene Forschung und Entwicklung in der Computer Science fördern wollte. Die Geschichte des Internets muss an dieser Stelle nicht erzählt werden. Wichtig ist nur festzuhalten, dass es niemals einen Masterplan für die Entwicklung des Internets gab, wie wir es heute kennen. Vielmehr konkurrierten bzw. koexistierten die auf den Internetprotokollen TCP/ 
IP basierenden Netze der Advanced Research Projects Agency (ARPANET) und das ab 1985 aufgebaute Netz der National Science Foundation (NSFNET) mit Wissenschaftsnetzen, die andere, auch kommerzielle Protokolle benutzten. Es erwies sich als unmöglich, die verschiedenen Forschungseinrichtungen, Regierungsagenturen, Netzträgerkonsortien und Privatfirmen auf einen gemeinsamen Standard für Wissenschaftsnetze festzulegen. Ein Versuch, privaten Netzbetreibern einen solchen Standard, hätte es ihn denn gegeben, ebenfalls nahe zu legen, wurde erst gar nicht in Betracht gezogen. Koordinationsgremien wie der Federal Networking Council waren zu schwach, um mehr als einen Informationsaustausch zwischen den Betreibern der großen öffentlichen Forschungsnetze zu ermöglichen. Mit der Entscheidung der National Science Foundation, das von ihr getragene Netz als Backbone für ein landesweites Wissenschaftsnetz auszulegen und es mit den TCP/IP Protokollen zu betreiben, war die organisatorische Nische geschaffen, aus der heraus sich die Protokolle zunächst im Bereich der Universitäten und öffentlichen Forschungseinrichtungen ausbreiteten (Werle 2000). Bezieht man in die Betrachtung noch die bereits gegen Ende der 1980er Jahre zahlreich vorhandenen kommerziellen Netze der Datenkommunikation, also die Unternehmensnetze, die öffentlichen und privaten Weitverkehrsnetze (WAN) sowie die lokalen Netze (LAN) mit ein, so erscheint die Situation in den USA geprägt durch technische Heterogenität und eine Vielfalt von Herstellern, Betreibern und Nutzern der Netze. Seit der Entflechtung des Telefonmonopolisten AT\&T im Jahre 1982 war auch der Telekommunikationssektor stärker wettbewerblich organisiert.

In den 1980er Jahren war die autonome Gestaltungsaufgabe und auch die Gestaltungsmöglichkeit nationaler Technologiepolitik noch wenig umstritten. In vielen Ländern Europas formierten sich Initiativen zum Aufbau landesweiter Wissenschafts- und Forschungsnetze, die sich untereinander, auch innerhalb der Europäischen Gemeinschaft, zunächst nur schwach koordinierten. In Deutschland ging die Initiative vom damaligen Bundesministerium für Forschung und Technologie (BMFT) aus. Es betrieb die Gründung des Vereins Deutsches Forschungsnetz (DFN), der sich 1982 konstituierte und zwei Jahre später seine Arbeit aufnahm. Die
Mitglieder stammten aus dem Hochschulbereich, der öffentlichen Großforschung und auch der Industrie. Als Selbsthilfeorganisation der Wissenschaft sollte der Verein eine Kommunikationsinfrastruktur schaffen, die bestehende kleinere regionale Netze integrieren, aber auch ein Gegengewicht gegen das sich in Westeuropa ausbreitende Wissenschaftsnetz EARN bilden sollte. EARN, das auf IBM Protokollen basierte und von der Firma auch gesponsert wurde, war an den Universitäten, die Zugang hatten, sehr beliebt. Mit dem DFN Verein verfolgte das BMFT gleichzeitig eine infrastruktur- und eine industriepolitische Zielsetzung. Letztere war auf die Stärkung der Wettbewerbsfähigkeit der deutschen Datenverarbeitungsindustrie gerichtet.

Zunächst hatte der Verein relativ wenige Mitglieder. Er agierte faktisch im Einvernehmen mit dem BMFT, das ihn maßgeblich finanzierte. Abgerundet wurde die Konzertierung durch die unumgängliche Mitwirkung der damaligen Deutschen Bundespost/ Telekom als Netzmonopolist. Der DFN Verein war als Verein ohne Netz auf die Bundespost angewiesen. Diese verfolgte zusammen mit den anderen europäischen Telekommunikationsmonopolisten und den führenden Firmen der europäischen DV-Industrie eine Politik der offenen Standards. Ziel war es, auf internationaler Ebene im Rahmen der zuständigen Standardisierungsorganisationen offene, d. h. nicht herstellergebundene (proprietäre) Standards für die Kommunikation in Datennetzen zu entwickeln. Mit diesen so genannten OSI Standards sollten Rechner aller Hersteller vernetzt werden und unterschiedlichste Dienste realisiert werden können. Der DFN Verein verpflichtete sich in seiner Satzung, die technischen Voraussetzungen für ein Wissenschaftsnetz auf der Basis solcher offener internationaler Standards zu schaffen (Leib und Werle 1997). Die Förderung von Forschungs- und Entwicklungsprojekten durch den Verein und das BMFT konzentrierte sich auf OSI. Bis etwa 1990 wurden technische Alternativen nicht nur nicht gefördert, sondern bekämpft (Zorn 1998, S. 200).

Anders als in den USA, wo die Regierung übrigens auch einige OSI Entwicklungen förderte, gelang es in Deutschland also, den relativ kleinen Kreis relevanter Akteure zu schließen und (fast) alles auf die Karte OSI zu setzen. Die „beste“ Technologie ist also nicht vom „Markt“ unter konkurrierenden Alternativen 
ausgewählt, sondern „politisch“ hierarchisch bestimmt worden, und zwar zu einem Zeitpunkt als sie sich noch in der Planung befand. So gesehen war technologiepolitische Steuerung durch ein kleines Netzwerk staatlicher und nichtstaatlicher Akteure erfolgreich. Allerdings hat sich OSI als ein Fehlschlag erwiesen. Ein Grund hierfür war die Komplexität des OSI Modells, die zur Folge hatte, dass es sehr lange dauerte, bis in den internationalen Organisationen der Standardisierung Standards für Teilkomponenten verabschiedet werden konnten. Dies wiederum führte zu langen Verzögerungen bei der Entwicklung von Produkten. So entstand zum Beispiel ein erster noch unzureichender Standard für Electronic Mail (X.400) 1984 und eine verbesserte, in der Praxis auch benutzte Version erst vier Jahre später. In der TCP/IP Welt wurde ein entsprechender Standard (SMTP) bereits 1982 veröffentlicht (RFC 821, 822), kurz bevor die TCP/IP Protokolle für das ARPANET verbindlich gemacht wurden (Schmidt und Werle 1998, S. 229-243).

Die Internetprotokolle TCP/IP sind zwar mit Forschungsmitteln aus dem Department of Defense entwickelt worden, sie waren aber weder geheim noch proprietär, sondern standen von Anfang an zur Implementation in Datennetzen kostenlos zur Verfügung. In dieser Hinsicht entsprachen sie also durchaus dem europäischen Postulat offener Standards. Doch nicht nur in Deutschland, sondern auch auf der Ebene der Europäischen Union hielt man noch in den frühen 1990er Jahren unbeirrt daran fest, TCP/IP zumindest nicht zu fördern. Die Kontrolle über die technischen Kommunikationsnetze lag immer noch weitgehend bei den kontinentaleuropäischen staatlichen Telekommunikationsunternehmen, die ihre eigenen Standards durchsetzen wollten. Angesichts der sich ankündigenden Liberalisierung der Telekommunikationsmärkte setzten sie auf die OSI-nahen ISDN Standards mit dem Ziel, alle möglichen Sprach- und Datendienste auf digitaler Basis in integrierter Form in einem Netz anbieten zu können und damit auch ihre Monopole zu verteidigen. Noch 1994 heißt es in den Empfehlungen „Europa und die globale Informationsgesellschaft“ der Bangemann-Kommission für den Europäischen Rat, dass ISDN als Baustein der Informationsgesellschaft gefördert werden müsse, während das Internet nur am Rande erwähnt wird (Werle
2002). Dies alles bestärkte die deutsche technologiepolitische Linie, was einen ihrer profilierten Kritiker, den Informatiker Werner Zorn, veranlasste, dem BMFT zu empfehlen: „Behaltet die Fördermillionen statt sie kontraproduktiv einzusetzen“ (VDI-Nachrichten vom 2.7.1999).

Ende der 1990er Jahre war das amerikanische NSFNET/Internet bereits privatisiert und weltweit etabliert. Schritt für Schritt setzte sich TCP/IP gegen andere Netzstandards, auch diejenigen großer privater Computerhersteller wie IBM durch. Nicht unbedingt einzelne etablierte Firmen der Branche, sondern insgesamt die amerikanische informationstechnische Industrie und die Anbieter Internet basierter Dienstleistungen profitierten von dem „First Mover Advantage“ der USA, der gegenüber Deutschland wegen der einseitigen Technologiepolitik besonders groß war.

Die Betrachtung der deutschen Internetpolitik bis Ende der 1990er Jahre zeigt, dass eine national ausgerichtete Politik durchaus möglich, wenn auch in diesem Falle nicht erfolgreich war. Politische Entscheidungen können ebenso wie unternehmerische Entscheidungen natürlich falsch sein, wobei es zumindest zu einem Teil von den strukturellen und institutionellen Bedingungen abhängt, wie folgenschwer Fehlentscheidungen sind. Die deutsche Situation mit einem Monopol in der Telekommunikation und einem relativ kleinen Kreis relevanter Organisationen im Politikfeld Internet hat es ermöglicht, die Förderung auf eine einzige technische Entwicklungslinie zu beschränken und sie langfristig vor der Konkurrenz anderer Optionen zu schützen. Dies war so in den USA gar nicht möglich. Es gab gute Gründe und gewichtige Interessen, die für die Förderung der von der deutschen Politik gewählten Variante sprachen, doch hätte eine größere Offenheit gegenüber konkurrierenden Varianten und die Berücksichtigung der Präferenzen der Nutzer sehr viel frühere Kursänderungen ermöglicht.

\section{Aus Fehlern lernen? Die neue Internetpo- litik}

War die verfehlte Internetpolitik speziell in den 1990er Jahren auch der Ignoranz relevanter Entscheidungsträger geschuldet, so darf doch nicht übersehen werden, dass die institutionellen Rahmenbedingungen ganz erheblich dazu 
beigetragen haben, dass diese Politik so lange durchgehalten werden konnte. Gegen Ende des letzten Jahrzehnts hatte sich dieser Rahmen dann jedoch insbesondere durch die Privatisierung der Deutschen Telekom und die Öffnung des Marktes für Dienste und Netze der Telekommunikation erheblich geändert. Die Liberalisierung der Telekommunikation stellt keinen deutschen Alleingang dar. Sie war vielmehr stark beeinflusst durch die lang anhaltenden strategischen Aktivitäten der EU-Kommission. Dennoch gibt es in diesem im Hinblick auf die Regulierungskompetenzen am stärksten europäisierten Wirtschaftszweig nationale Gestaltungsspielräume, die auch genutzt werden (Schneider und Tenbücken 2004).

Im Zusammenhang mit der Liberalisierung sind nicht nur die Preise für Produkte und Dienste der Telekommunikation deutlich gesunken, sondern, wie die Auflösung des für diesen Bereich zuständigen Ministeriums zeigt, auch fest gefügte Strukturen der internetpolitischen Konzertierung in Bewegung geraten. Gleichzeitig wurden die Folgen der Politik der beiden vergangenen Jahrzehnte deutlich. Ende der 1990er Jahre gab es keine für den Weltmarkt relevanten deutschen Firmen im eine erhebliche Dynamik aufweisenden Internetgeschäft - weder bei der Hardware noch bei der Software noch bei den Dienstleistungen. Auch in der Nutzung des Netzes hinkte Deutschland im internationalen Vergleich deutlich hinterher. Daran konnten auch die Initiativen einiger Bundesländer, des BMWI und des BMFT zur Förderung von Multimedia seit Mitte der 1990er Jahre nicht viel ändern. „Deutschlands Weg in die Informationsgesellschaft“ führte nicht über das Internet, sondern emulierte das von der Bangemann-Kommission entworfene europäische Modell. Die Förderung war techniklastig und industrielastig und $\mathrm{zu}$ wenig an den Nutzern orientiert (Werle 2002).

Unterschiedliche Quellen zeigen, dass Deutschland im Jahre 2000 im Hinblick auf die berufliche und private Nutzung des Internets deutlich hinter vielen Industriestaaten zurück lag. Im vom Bundesministerium für Bildung und Forschung (BMBF) veröffentlichten Bericht zur technologischen Leistungsfähigkeit 2002 resümieren die Autoren, dass Deutschland bei den meisten Indikatoren der Diffusion von IuK-Technologien ,nicht so schnell voran- gekommen“ sei wie die meisten anderen Länder, sondern „unbemerkt im hinteren Mittelfeld“ rangiere (BMBF 2003, S. 97). Dies gilt besonders für das Internet.

Dort, wo wie in der Wissenschaft die Nutzer Einfluss nehmen konnten, waren am frühesten Kursänderungen $\mathrm{zu}$ verzeichnen. So nahm der DFN Verein Anfang der 1990er Jahre ein schmalbandiges Wissenschaftsnetz (WIN) in Betrieb, auf dem auch Internet Dienste angeboten wurden. Diese Dienste entwickelten sich sehr erfolgreich mit der Folge, dass der Verein hohe Einnahmen erzielte, die für den weiteren Ausbau von Netzen und Diensten verwendet wurden. Hier ist es bis Ende der 1990er Jahre gelungen, Anschluss an die internationale Entwicklung zu gewinnen. Heute betreibt das Network Operating Center des Vereins das Gigabit-WIN mit dem DFNInternet Dienst als dem wichtigsten.

Das Beispiel der amerikanischen Wirtschaft, deren Wachstumsgewinne in der zweiten Hälfte der 1990er Jahre vor allem auf die schnelle und gründliche Verbreitung einer modernen IuK-Infrastruktur zurückgeführt werden kann (BMBF 2003, S. 91), und auch die Erfahrungen mit dem deutschen Wissenschaftsnetz haben eine deutliche Kursänderung der deutschen Internetpolitik angestoßen. Parallel hierzu lässt sich auch auf der Ebene der EU eine Umorientierung beobachten. Im Rahmen ihrer im März 2000 beschlossenen $e$ Europe Initiative (COM (99) 687) legt die EU nun besonderes Gewicht auf die Förderung des Zugangs zum Internet und dessen Nutzung in vielen gesellschaftlichen Bereichen. Viele deutsche Initiativen sind denjenigen der EU ähnlich, ohne dass Deutschland lediglich die EU-Vorgaben ausführt.

Bemerkenswert ist die Betonung einer leistungsfähigen technischen Kommunikationsinfrastruktur als Voraussetzung für die Wettbewerbsfähigkeit der deutschen Wirtschaft. Ziel der öffentlichen Förderung von Forschung und Entwicklung ist es nicht mehr, die Weltmärkte mit deutscher Technologie zu erobern, sondern „mit dazu beizutragen, dass die neuen Technologien und Anwendungen in Deutschland erforscht, entwickelt und genutzt werden“" (BMWA 2002, S. 56). Zugang zum Internet und Nutzung der Dienste sowie die darauf bezogenen Forschungs- und Entwicklungsaktivitäten 
werden mit einer Vielzahl eher kleinerer Projekte gefördert. An der Förderung sind unterschiedliche Ministerien beteiligt. Einbezogen ist auch als prominentestes Beispiel einer Public Private Partnership in diesem Bereich die „Initiative D21“. Dieser 1999 gegründete Verein koordiniert unterschiedliche private und öffentliche Einrichtungen in ihrem Bemühen, den Umgang mit den modernen Informations- und Kommunikationstechniken zu vermitteln und auch die Rahmenbedingungen ihrer Ausbreitung und Nutzung zu verbessern. Die Schaffung adäquater rechtlich-institutioneller Rahmenbedingungen für die effiziente und sichere Nutzung des Internets wird als ebenso wichtig wie die technologiebezogene Förderung eingeschätzt. Im „Aktionsprogramm Informationsgesellschaft 2006“ der Bundesregierung nimmt die Gestaltung dieser Rahmenbedingungen wie Datenschutz, Schutz geistigen Eigentums oder die Eindämmung von Spam Mails einen breiten Raum ein (BMWA und BMBF 2003). Erstmalig wird im Bundesforschungsbericht 2004 das Internet neben Multimedia als eigenständiger Förderbereich aufgeführt und die Vorreiterrolle des Forschungsnetzes bei innovativen Internetanwendungen betont (BMBF 2004, S. 277).

Bemerkenswert ist die Zurückhaltung der deutschen Politik dort, wo es um die Koordination der Grundfunktionen des globalen Internets geht. Die Sicherung von Interoperabilität und Kompatibilität vor allem durch globale Standards, die Gewährleistung von Interkonnektivität im Sinne des gesicherten Zugangs zum Netz und der Zusammenschaltung von Teilnetzen sowie die einheitliche Adressierung und Identifikation der Teilnehmer werden ganz überwiegend als Selbstregulierungsaufgabe privater Organisationen angesehen oder dem Markt überlassen (vgl. Werle 2000). Dies gilt grundsätzlich auch für den nationalen Rahmen, wo z. B. eine Genossenschaft (DENIC) und keine staatliche Agentur die Top Level Domain .de verwaltet. Es fragt sich allerdings auch, ob es einer einzelnen Regierung möglich wäre, in die Koordination der Grundfunktionen des globalen Internets steuernd einzugreifen und wie weit über Public Private Partnership hinausgehende politische Einflüsse zweckmäßig oder gar notwendig sind.

Etwas anders ist die Situation auf der Ebene der Anwendungen und Dienste des Inter- nets. Auch hier ist es für die Regierungen schwierig, Regulierungen durchzusetzen. Doch verlassen sie sich nicht nur auf Selbstregulierung, sondern sind unverkennbar bemüht, auf multilateraler Basis und gelegentlich auch im Alleingang dem jeweiligen Regulierungsrahmen Geltung zu verschaffen (Holznagel und Werle 2002). Dies ist auch notwendig, vor allem um das Vertrauen in die Sicherheit der Nutzung des Netzes zu stärken. Dabei sind die Durchsetzungsmöglichkeiten einer einzelnen Regierung umso besser, je größer der nationale Internetmarkt ist. Die Förderung des Zugangs zum und der Nutzung des Internets durch die deutsche Politik wird also, wenn sie erfolgreich ist, auch die nationalen Steuerungsmöglichkeiten verbessern. Dies garantiert aber nicht in jedem Fall auch einen positiven Steuerungseffekt, wie ja gerade die deutsche Erfahrung in den frühen Jahren des Internets gezeigt hat.

Man hat jedoch aus den Fehlern gelernt. Die zuständigen Ministerien verweisen darauf, dass der Rückstand zu den führenden Ländern speziell in der Häufigkeit und Breite der Nutzung des Internets deutlich verringert wurde. In einem auf mehreren Indikatoren basierenden „Digital Access Index“ der Internationalen Telekommunikationsunion aus dem Jahre 2002 befindet sich Deutschland in der Spitzengruppe mit „High Access“, wenn auch erst auf Platz 18. Auch sind praktisch alle deutschen Schulen an das Internet angeschlossen. Allerdings rangiert Deutschland mit vier Interanschlüssen je 100 Sekundarschüler auf einem hinteren Platz nur vor Portugal und Griechenland (Grupp, Legler und Licht 2004, S. 59).

Es gibt also noch erhebliche Defizite, weil die „Globalisierung keine Gleichschaltung der Innovationssysteme“ bringt (ebd., S. 91). Deshalb kann es auch keinen Königsweg geben, den alle nationalen Internetpolitiken zu beschreiten haben. Bei aller Gefahr einer allzu starken Fragmentierung erscheint es aber doch nützlich, dass die Zuständigkeit für die Internetpolitik bei gleichzeitig offenen Grenzen national breit verteilt ist. Während ersteres rein national bestimmt ist, verdankt sich die Offenheit der Grenzen auch der Europäisierung und Globalisierung. In jedem Fall wird die Tragweite von politischen Fehlentscheidungen durch diese Entwicklungen reduziert. 


\section{Literatur}

Blumenthal, M.S.; Clark, D.D., 2001: Rethinking The Design Of The Internet: The End-To-End Arguments Vs. The Brave New World. In: Compaine, B.M.; Greenstein, S. (eds.): Communications Policy in Transition. The Internet and Beyond. Cambridge, MA: MIT Press, S. 91-139

BMBF - Bundesministerium für Bildung und Forschung (Hrsg.), 2003: Zur technologischen Leistungsfähigkeit Deutschlands 2002. Bonn: BMBF

BMBF (Hrsg.), 2004: Bundesbericht Forschung 2004. Bonn: BMBF

BMWA - Bundesministerium für Wirtschaft und Arbeit (Hrsg.), 2002: Informationsgesellschaft Deutschland. Fortschrittsbericht zum Aktionsprogramm der Bundesregierung. Berlin: BMWA

BMWA; BMBF (Hrsg.), 2003: Aktionsprogramm Informationsgesellschaft Deutschland 2006. Berlin: BMWA, BMBF

Grande, E., 1996: Das Paradox der Schwäche: Forschungspolitik und die Einflußlogik europäischer Politikverflechtung. In: Jachtenfuchs, M.; KohlerKoch, B. (Hrsg.): Europäische Integration. Opladen: Leske + Budrich, S. 373-399

Grupp, H.; Legler, H.; Licht, G., 2004: Technologie und Qualifikation für neue Märkte. Bonn: BMBF

Holznagel, B.; Werle, R., 2002: Sectors und Strategies of Global Communications Regulation. In: Zeitschrift für Rechtssoziologie 23, S. 3-23

Leib, V.; Werle, R., 1997: Wissenschaftsnetze in Europa und den USA. Die Rolle staatlicher Akteure bei ihrer Bereitstellung. In: Werle, R.; Lang, C. (Hrsg.): Modell Internet? Entwicklungsperspektiven neuer Kommunikationsnetze, Frankfurt a. M.: Campus, S. 157-185

Lemley, M.A.; Lessig, L., 2004: The End of End-ToEnd. Preserving the Architecture of the Internet in the Broadband Era. In: Cooper, M.N. (ed.): Open Architecture as Communications Policy. Preserving Internet Freedom in the Broadband Era. Center for Internet and Society, Stanford Law School, S. 41-92

Scharpf, F., 1999: Regieren in Europa. Effektiv und demokratisch? Frankfurt a. M.: Campus

Schmidt, S.K.; Werle, R., 1998: Coordinating Technology. Studies in the International Standardization of Telecommunications. Cambridge, MA: MIT Press

Schneider, V.; Tenbücken, M. (Hrsg.), 2004: Der Staat auf dem Rückzug. Die Privatisierung öffentlicher Infrastrukturen. Frankfurt a. M.: Campus

Simonis, G., 2004: Nachhaltige Technologiepolitik Möglichkeiten und Grenzen politischer Steuerung. Manuskript, Hagen
Streeck, W., 2004: Globalisierung: Mythos und Wirklichkeit. MPIfG Working Paper 04/4, MaxPlanck-Institut für Gesellschaftsforschung, Köln; http://www.mpi-fg-koeln.mpg.de/pu/workpap/ wp04-4/wp04-4.html

Werle, $R$., 2000: Innovationspotentiale im Internet Selbstregelung auf Strukturebene. In: HoffmannRiem, W. (Hrsg.): Innovation und Telekommunikation. Rechtliche Steuerung von Innovationsprozessen in der Telekommunikation. Baden-Baden: Nomos, S. 141-160

Werle, R., 2001: Liberalisierung und politische Techniksteuerung. In: Politische Vierteljahresschrift, Sonderheft 31/2000: Politik und Technik. Analysen zum Verhältnis von technologischem, politischem und staatlichem Wandel am Anfang des 21. Jahrhunderts, S. 407-424

Werle, R., 2002: Internet@Europe: Overcoming institutional fragmentation and policy failure. In: Jordana, J. (ed.): Governing Telecommunications and the New Information Society in Europe. Cheltenham: Edward Elgar, S. 137-158

Zorn, W., 1998: Verfehlte Entwicklung. Telekommunikationspolitik in Deutschland. In: Leggewie, C.; Maar, C. (Hrsg.): Internet \& Politik. Von der Zuschauer- zur Beteiligungsdemokratie. Köln: Bollmann, S. 194-206

\section{Kontakt}

Dr. Raymund Werle

Max-Planck-Institut für Gesellschaftsforschung

Paulstraße 3, 50676 Köln

Tel.: +49 (0) 221 / 2767224

Fax: +49 (0) 221 / 2767452

E-Mail: we@mpifg.de

Internet: http://www.mpifg.de/people/we/ 